\title{
Avaliação de procedimentos urbanos de descarte de resíduos eletroeletrônicos domiciliares: uma proposta metodológica
}

Evaluation of urban procedures for household electrical and electronic waste disposal: a methodological proposal

Evaluación de procedimientos urbanos de descarte de residuos electro electrónicos domésticos: una propuesta metodológica.

\section{Claudia Magalhaes Khair Cunha Mestranda, UFRJ, Brasil. Claudia.khair@poli.ufrj.br}

\section{Claudio Fernando Mahler} Professor Doutor, UFRJ, Brasil. cfmahler@gmail.com

Elen Beatriz Acordi Vasques Pacheco Professora Doutora, UFRJ, Brasil. elen@ima.ufrj.br 


\begin{abstract}
RESUMO
O papel do consumidor na cadeia pós-consumo de resíduos, inclusive eletroeletrônicos, é considerado, em diversos países, essencial para a implantação da economia circular. No Brasil, tal participação é ressaltada na Política Nacional de Resíduos Sólidos. No entanto, na ausência de um sistema efetivo de logística reversa de resíduos de equipamentos eletroeletrônicos (REEE) que defina procedimentos adequados de descarte, o consumidor adota seus próprios métodos - descarte junto ao lixo comum, em terreno baldio, empresa informal, ou simplesmente manter em casa impactando todo o sistema de gestão deste resíduo. Este trabalho visa propor uma metodologia para investigar como os cidadãos de determinada localidade vêm realizando o descarte de REEE existentes em seu domicílio, objetivando conhecer os principais hábitos de descarte e motivações, e relacioná-los a aspectos socioeconômicos através do Índice de Desenvolvimento Humano (IDH) da localidade selecionada. Esta metodologia permite ainda identificar possíveis problemas, facilidades e dificuldades no descarte de REEE enfrentados pela população, colaborando no desenvolvimento de sugestões para aumentar a probabilidade de retorno dos REEE ao setor produtivo, visando sua reutilização, reciclagem ou outra forma de destinação final correta. Exemplos práticos de aplicação da metodologia para definição dos locais a serem estudados e do questionário a ser aplicado são também aqui apresentados. Esperase que os resultados obtidos e as conclusões permitam sugerir ações de melhorias, especialmente através de políticas públicas como o acordo setorial, contribuindo com avanços na gestão dos REEE no país.
\end{abstract}

PALAVRAS-CHAVE: Resíduo Eletroeletrônico. Descarte de REEE. Consumidor.

\title{
SUMMARY
}

The role of consumers in the post-consumer waste chain, including electrical and electronic equipment, is considered, in many countries, essential for the implementation of the circular economy. In Brazil, such participation is highlighted in the National Solid Waste Policy. However, in the absence of an effective reverse logistics system for waste electrical and electronic equipment (WEEE) to establish proper disposal procedures, the consumers adopt their own methods disposing along with ordinary garbage, on wasteland, informal business, or simply keeping at home - impacting the entire waste management system. This paper intends to propose a methodology to investigate how citizens of a given locality have been disposing their household WEEE, aiming to know the main disposal habits and motivations, and relate them to socioeconomic aspects through the Human Development Index (HDI) of the selected location. This methodology also helps to identify possible problems, ease and difficulties faced by the population regarding WEEE disposal, while collaborating with the development of suggestions to increase the likelihood of WEEE return to the productive sector, focusing on its reuse, recycling or other form of correct final destination. Practical examples of how to apply the methodology to define the places to be studied and the questionnaire to be applied are also presented here. The results and conclusions are expected to support improvement propositions, especially through public policies such as the sector agreement, contributing to the development of the WEEE management in the country.

KEYWORDS: Electroelectronic waste. Waste disposal. Consumer.

\section{RESUMEN}

El papel de los consumidores en la cadena de residuos posconsumo, incluidos los electro electrónicos, se considera, en muchos países, esencial para la implementación de la economía circular. En Brasil, dicha participación se destaca en la Política Nacional de Residuos Sólidos. Sin embargo, en ausencia de un sistema eficaz de logística inversa de residuos de aparatos electro electrónico (RAEE) que defina los procedimientos adecuados de descarte, el consumidor adopta sus propios métodos: eliminar con los desechos normales, en terrenos baldíos, negocios informales o simplemente mantener en casa, impactando todo el sistema de gestión de residuos. Este documento tiene como objetivo proponer una metodología para investigar cómo los ciudadanos de una localidad determinada han estado eliminando los RAEE existentes en sus hogares, con el objetivo de conocer los principales hábitos y motivaciones de eliminación, y relacionarlos con los aspectos socioeconómicos a través del Índice de Desarrollo Humano (IDH) de la ubicación seleccionada. Esta metodología también permite identificar posibles problemas, facilidades y dificultades que enfrenta la población para el descarte de RAEE, colaborando en el desarrollo de sugerencias para aumentar la probabilidad de retorno de RAEE al sector productivo, con el objetivo de su reutilización, reciclaje u otra forma de destino final correcto. Aquí también se presentan ejemplos prácticos de la aplicación de la metodología para definir los lugares a estudiar y el cuestionario a aplicar. Se espera que los resultados y conclusiones sugieran mejoras, especialmente a través de políticas públicas como el acuerdo sectorial, contribuyendo a los avances en la gestión de RAEE en el país.

PALABRAS CLAVE: Residuos electroelectrónicos. Eliminación de residuos. Consumidor. 


\section{Periódica Eletranica}




\section{INTRODUÇÃO}

A economia circular é apontada como uma alternativa ao modelo tradicional de produção e consumo que busca o crescimento contínuo e, para tal, absorve crescentes volumes de recursos naturais. Dentro desse conceito, estudos vêm sendo publicados, especialmente na China e na União Europeia, para estabelecer as bases deste novo conceito, e compreender como implementá-lo (Ghisellini, 2016).

De acordo com Su et al. (2013), países como Alemanha e Japão foram pioneiros na adoção de políticas públicas para implantar a economia circular através da gestão integrada de resíduos sólidos, visando evitar tanto a degradação ambiental como também a escassez de recursos materiais importantes. Como observado nestes países, a participação do consumidor é indispensável (Su et al., 2013). O consumidor tem um papel fundamental ao comprar de forma consciente, promover o uso e o reuso pelo tempo máximo de vida útil do produto e ainda efetuar o descarte mais adequado. O ciclo virtuoso proposto pela economia circular ganha impulso com o aumento da participação do consumidor (Ghisellini, 2016).

Dentre os diversos tipos de resíduos produzidos no mundo, chama a atenção o crescente aumento na geração de resíduos eletroeletrônicos (REEE). Em 2016, foram gerados 44,7 x $10^{6}$ toneladas de REEE no planeta, representando cerca de 6,1 kg per capita (Baldé et al, 2017). Em 2014, haviam sido 41,8 × 106 t, ou seja, $5.9 \mathrm{~kg} / \mathrm{hab}$ (Baldé et al., 2015). Nas duas últimas décadas, tal volume crescente vem alarmando os estudiosos e responsáveis pela gestão de resíduos em diversos países. Políticas públicas específicas para o setor que estimulam a produção mais limpa, o eco-design e os sistemas de logística reversa para reciclagem vem sendo desenvolvidas e implantadas, e reforçam, entre outros aspectos, a responsabilidade compartilhada do consumidor na fase de coleta de REEE para reciclagem.

Dentro do conceito de economia circular, o consumidor consciente, ao optar por um produto ou serviço mais sustentável, exerce uma função crucial no momento da compra (Zhijun e Nailing, 2007; Geng e Doberstein, 2008; Su et al., 2013 apud Ghisellini, 2016). Além disso, após a aquisição e o uso, o consumidor torna-se responsável por encaminhar o produto adequadamente para reuso, reparo ou reciclagem. Portanto, o consumidor representa um importante elo no sistema de logística reversa de resíduos de equipamentos eletroeletrônicos (Nnorom et al., 2009; Yin et al, 2013).

Na Europa, para estimular a participação dos consumidores no sistema de logística reversa de REEE, a Diretiva Europeia estabelece que eles podem retornar seus equipamentos sem custo, através dos distribuidores, que são obrigados a recebê-los (Aizawa, 2008; European Union, 2012).

No Japão, as políticas públicas voltadas à economia circular e logística reversa de REEE reforçam a responsabilidade do consumidor. Há um sistema de manifesto que registra toda a logística de devolução do equipamento pelo consumidor à um revendedor, o transporte até o fabricante ou importador, e ao reciclador. Além disso, o consumidor é o responsável financeiro pela coleta e reciclagem. Ele paga uma taxa ao descartar o equipamento (Aizawa, 2008).

$\mathrm{Na}$ China, alguns estudos sobre a implantação da economia circular no país defendem a adoção de políticas de incentivo ao consumo sustentável e de responsabilidade compartilhada do consumidor quanto à reciclagem de produtos pós-consumo (Feng e Yan, 2007). 
No Brasil, a Política Nacional de Resíduos Sólidos (Brasil, 2010) adota a responsabilidade compartilhada entre produtores e consumidores, e também destaca a importância do papel do consumidor nos sistemas de logística reversa. $\mathrm{O}$ acordo setorial para implantação do sistema de logística reversa para o setor de REEE teve seu edital lançado pelo Ministério do Meio Ambiente (MMA) em 2013. Uma proposta única foi sintetizada em 2014 a partir de várias sugestões recebidas. Longamente discutida pelo setor, a proposta resultante foi finalmente encaminhada para consulta pública em agosto de 2019 (Acordos Setoriais, MMA). Após receber os comentários de todos os stakeholders, incluindo a sociedade civil, o acordo setorial está atualmente em fase de revisão e deve ser assinado em breve.

\section{A IMPORTÂNCIA DO CONSUMIDOR NO PROCESSO DE RECICLAGEM DE REEE}

A área de resíduos no Brasil é uma preocupação crescente, em especial no tocante aos REEE. É preciso melhorar a compreensão sobre um elo fundamental da cadeia de resíduos eletroeletrônicos: o consumidor.

A logística reversa começa pelo consumidor (Souza, Paula, e Souza-Pinto, 2012). É o consumidor (residencial ou comercial) que transforma o equipamento eletroeletrônico em resíduo, e é o mesmo consumidor o responsável por definir inicialmente o caminho que este resíduo vai seguir, seja o aterro sanitário ou a reciclagem (Dias et al., 2017).

Em um sistema de logística reversa de REEE, se estipula detalhadamente os critérios de coleta e transporte deste tipo de resíduo; a cobertura geográfica e as formas de coleta (pontos de entrega, centrais de recebimento fixas ou itinerantes); os tipos de destinação dada aos REEE; as formas de participação do consumidor; e os mecanismos de divulgação destes procedimentos aos consumidores. A maioria dos sistemas de logística reversa de REEE se apoia no princípio que o consumidor vai adotar novos hábitos, separar seu resíduo e entrega-lo nos pontos de coleta. Sem estrutura, educação e informação adequadas, a participação do público será dificultada (Darby e Obara, 2005).

Sem conhecer com clareza o sistema de logística reversa, o consumidor acaba por adotar diferentes atitudes no momento de descartar seus resíduos eletroeletrônicos, tais como envio para reutilização ou para reciclagem em empresa não regulamentada, descarte inadequado junto ao lixo comum, entre outros (COMLURB, 2017; Rodrigues, Gunther e Boscov, 2015).

Apesar da dificuldade na obtenção de dados relativos ao setor - formal e informal - brasileiro de reciclagem de REEE, estudos mostram os diferentes procedimentos de descarte da população. De acordo com Rodrigues, Gunther e Boscov (2015), existe a:

Prática dos indivíduos de guardarem equipamentos obsoletos e avariados em suas residências por tempo indeterminado devido à carência de opções de destinação e também por esperarem uma oportunidade futura para obter algum retorno financeiro por um bem no qual houve um investimento inicial importante. (RODRIGUES, Gunther e Boscov, 2015, p.438).

Em função disso, os autores mensuraram em seu estudo, através de questionário, quantidades e tipos de resíduos eletroeletrônicos domiciliares não descartados pelos habitantes da cidade de São Paulo. No entanto, não foram consideradas características socioeconômicas da população estudada. 
Em seu estudo, Echegaray e Hansstein (2017) investigaram, também através de questionários, a intenção da população brasileira em coletar REEE versus a sua real atitude frente ao descarte, constatando uma enorme distância entre intenção e ação. A pesquisa foi aplicada em 9 das principais cidades brasileiras e foram considerados os aspectos socioambientais, como gênero, idade, renda e nível de escolaridade.

De acordo com Dias et al. (2017), existiam no Brasil, em 2017, 134 unidades produtivas que efetivamente trabalhavam com REEEs. Estas unidades informaram que as três principais formas de obtenção de material eram: parcerias com empresas para destinação correta de seus equipamentos obsoletos ( $81,8 \%)$; coleta pulverizada de REEEs, usando frota própria $(68,2 \%)$; e material entregue pela população diretamente nos Pontos de Entrega Voluntária (PEVs) das unidades produtivas $(63,6 \%)$. Portanto, observa-se que mais da metade das unidades produtivas de REEE brasileiras contam com o material depositado pelas pessoas nos PEVs, evidenciando que o comportamento da população frente ao descarte de REEEs impacta diretamente a cadeia de reciclagem pós-consumo deste tipo de material.

Conhecer e compreender as razões que levam o consumidor a descartar - ou a reter - possibilita a adoção de medidas de estímulo ao descarte correto. O aumento do volume depositado nos PEVs pode beneficiar a cadeia de reciclagem de eletroeletrônicos sob aspectos econômicos, pois a logística de coleta nos PEVs apresenta melhor custo-benefício, por se tratar de rota prédefinida e com melhor qualidade de material (Bernardo e Lima, 2015). No âmbito social, o maior volume recebido pode impulsionar a geração de empregos.

Estudos científicos constatam a existência de uma parcela de REEE armazenada em residências no Brasil (Rodrigues, Gunther e Boscov, 2015; Echegaray e Hansstein, 2017). Por outro lado, algumas análises constatam que parte dos REEE são descartados incorretamente junto ao resíduo doméstico comum, indo para aterros (COMLURB, 2017).

É importante, portanto, examinar como o consumidor urbano age, na ausência do acordo setorial e outras políticas públicas de REEE, diante da questão do descarte dos resíduos eletroeletrônicos que estão sob sua responsabilidade em seu domicílio, e contribuir para a geração de dados primários sobre a retenção sistemática de REEE nas residências. $O$ conhecimento obtido pode vir a colaborar no estabelecimento de procedimentos diferenciados no acordo setorial de logística reversa de REEE, incentivando a participação da sociedade na cadeia de reciclagem deste material e colaborando para o aumento de REEE corretamente descartado no Brasil.

Neste artigo, pretende-se propor uma metodologia que permita investigar como o cidadão realiza o descarte de REEE gerado em seu domicílio, no intuito de conhecer as principais razões que o motivam a reter ou o impedem de descartar seus resíduos eletroeletrônicos, ou mesmo destiná-los. A metodologia proposta tem como objetivo identificar as motivações e relacionálas a aspectos socioeconômicos da população através do Índice de Desenvolvimento Humano (IDH) de cada localidade selecionada.

Além da investigação, identificação e análise dos hábitos de descarte de resíduos de equipamentos eletroeletrônicos de uma determinada população, esta metodologia colabora na identificação de possíveis problemas, facilidades e dificuldades enfrentadas pela população neste elo da cadeia pós-consumo dos REEE e eventualmente poderá colaborar no 
desenvolvimento de sugestões para aumentar a probabilidade de retorno dos REEE ao setor produtivo, visando à reutilização, reciclagem ou outra forma de destinação final correta.

\section{A METODOLOGIA PROPOSTA PARA A AVALIAÇÃO DO DESCARTE}

Propõe-se estudar os procedimentos adotados pela população de dois bairros de um município, em diferentes situações socioeconômicas, com relação aos resíduos de equipamentos eletroeletrônicos. Deverão ser observados os aspectos voltados à retenção - quantidades, período e possíveis razões -, verificando-se as opções existentes de descarte destes resíduos e as razões que levam a população a optar por diferentes formas de descarte.

Este tipo de estudo é de natureza quantitativa, ou seja, deve ser efetuado por meio de aplicação de questionário com perguntas cujas respostas podem ser traduzidas em números, ou seja, dados primários que serão quantificados e analisados estatisticamente. Segundo Marconi e Lakatos (2011), a pesquisa quantitativa "é a mais apropriada para apurar atitudes e responsabilidade dos entrevistados, uma vez que emprega questionários". Os questionários deverão ser aplicados em dois bairros da cidade pesquisada. A proposta metodológica sugere a utilização de escolas do bairro como facilitadores da pesquisa pois através delas os questionários chegarão aos familiares dos alunos e posteriormente retornarão.

Esta proposta de pesquisa pode ser classificada como descritiva e explicativa quanto aos objetivos, uma vez que além de efetuar um levantamento bibliográfico sobre o tema, também busca explicar como se dá o processo de descarte efetuado pela população estudada. Quanto aos meios utilizados, classifica-se como pesquisa bibliográfica e de campo, incluindo um estudo de caso. No que se refere à abordagem temporal, poderá ser classificada com pesquisa de corte transversal, pois os dados serão coletados em um determinado momento no tempo, e não através da observação contínua, evolutiva, do comportamento da população ao longo do tempo (Ramalho e Marques, 2009; Birochi, 2015).

\subsection{Definição dos locais a serem estudados}

$\mathrm{O} I \mathrm{IH}$, indicador sugerido para ser utilizado nesta metodologia, foi adotado pela ONU a partir de 1993, em seu Programa das Nações Unidas para o Desenvolvimento (PNUD), por proporcionar uma forma de medir o desenvolvimento humano de cada país pelo bem estar de sua população, e não apenas pelo Produto Interno Bruto (PIB) per capita (Neumayer, 2001)

O IDH é calculado a partir de três componentes socioeconômicas relativas à população de um país: saúde, renda e educação. Uma vida longa e saudável é representada pela expectativa de vida ao nascer; a componente econômica é medida pelo PIB per capita; e a educação reflete o acesso ao conhecimento que é proporcionado à população, representado pelo grau de escolaridade média de adultos acima de 25 anos e pela expectativa de anos na escola para crianças em idade escolar (UNDP, 2016).

A definição dos dois bairros a serem pesquisados deverá ser produto do cruzamento dos dados de duas fontes de pesquisa distintas. Uma delas deverá conter a relação bairro x gravimetria dos resíduos, ou seja, apresentar, para cada bairro da cidade, as informações gravimétricas sobre os resíduos ali produzidos, incluindo REEE. A outra fonte deverá conter a relação bairro x IDH. Desta forma, será possível identificar dois bairros com diferentes IDHs e obter informações sobre a geração de REEE de cada um. 
Ao propor a análise do descarte em dois territórios extremos da cidade, em termos de IDH, espera-se que os resultados obtidos neste estudo permitam estabelecer um intervalo representativo para a análise sobre retenção e descarte de REEE no município estudado.

\subsection{Definição da amostra mínima}

A metodologia aqui apresentada propõe a realização de uma pesquisa quantitativa através da aplicação de questionários. Para que esta pesquisa tenha valor científico, precisa ser aplicada em uma amostra estatística com significativa representatividade, ou seja, que represente um determinado universo de pessoas, para que se possa posteriormente extrapolar os resultados para aquele ambiente (Marconi e Lakatos, 2011). Com base na população do local escolhido para a pesquisa, deve-se calcular o número mínimo de questionários respondidos necessários para que se alcance o grau de confiança desejado.

Nesta pesquisa, recomenda-se adotar um grau de confiança de $95 \%$ e erro amostral de $5 \%$. A população $\mathrm{P}$ da pesquisa é formada pelas populações P1 (bairro 1) e P2 (bairro 2). Utilizando-se tratamento estatísticos a partir destes dados, tais como média e desvio padrão, pode-se calcular o tamanho da amostra, ou seja, a quantidade mínima n1 e n2 de questionários que deverão ser respondidos respectivamente em cada bairro.

\subsection{Desenvolvimento do questionário a ser aplicado}

O questionário a ser aplicado deve ter foco no objetivo principal deste trabalho, ou seja, deve ser construído de forma a permitir a identificação e avaliação dos hábitos de descarte de resíduos de equipamentos eletroeletrônicos de uma determinada população, conforme recomendado por Günther (2003). O mesmo questionário deverá ser aplicado em ambos os bairros definidos para a pesquisa. É preciso estabelecer com clareza o propósito de cada pergunta incluída, e o significado de cada resposta para a pesquisa.

Uma revisão bibliográfica preliminar é recomendada, efetuando o levantamento, seleção, leitura e análise de artigos técnicos referentes a metodologias de construção de questionários e entrevistas, em especial os questionários comportamentais e os relacionados aos REEE. Este material auxiliará na elaboração das perguntas, para que se possa obter, com isenção e sem influências, respostas autênticas das pessoas consultadas, e um número suficiente de questionários respondidos (Günther, 2003).

Um desafio que se apresenta é o fato da população-alvo do questionário ser formada por dois grupos com diferenças socioeconômicas consideráveis. O questionário e as formas de abordagem devem levar tais diferenças em conta, pois elas podem interferir no resultado. 0 próprio linguajar deve ser cuidadosamente pensado. Segundo Günther (2003), deve-se evitar gírias, abreviações e termos sofisticados que possam comprometer a compreensão das perguntas pelo respondente ou gerar ambiguidade.

A sequência das perguntas também é importante, pois facilita a compreensão e, consequentemente, o raciocínio do respondente, reduzindo o tempo de resposta total, o que aumenta as chances de obter um questionário completamente respondido. No caso do questionário deste estudo, as perguntas deverão ser ordenadas numa sequência que inicia estimulando o respondente a perceber o problema, passa às motivações e segue em direção à possíveis ações mitigadoras nas quais ele poderia se envolver. De acordo com cada resposta, será possível identificar a atitude de descarte adotada pelo respondente em cada situação 
apresentada. As perguntas que pretendem aferir atitudes demandam certa preparação inicial, esclarecendo, por exemplo, o objeto das atitudes (Günther, 2003). O questionário desta pesquisa deverá apresentar, em seu cabeçalho, uma lista sucinta de exemplos de aparelhos elétricos e eletrônicos de pequeno porte que, ao fim de sua vida útil, são considerados REEE.

Vale comentar que as características socioeconômicas das populações entrevistadas neste estudo já estarão definidas pelo IDH de cada uma. Não há, portanto, necessidade do questionário apresentar uma seção adicional de perguntas socioeconômicas, o que toma tempo e muitas vezes gera constrangimento ao respondente.

\subsection{Validação do questionário}

Uma vez definido o tipo, o formato, a quantidade e a redação de cada pergunta do questionário, é importante validá-lo, de modo a comprovar que o instrumento é capaz de mensurar o que ele se propõe a medir. Esta etapa inclui a realização de um teste piloto com público reduzido para validação de conteúdo, validação da construção, confiabilidade, validade de critério e consistência interna do questionário (Hoss e Caten, 2010). Este piloto permitirá ainda testar se há necessidade de incluir, excluir ou melhorar a redação de alguma questão e se a forma de comunicação (via escola) funcionará conforme o esperado.

Caso seja identificada alguma falha, é importante avaliar se o questionário deve ser modificado ou não, e proceder com as eventuais adequações, antes de sua aplicação na sua totalidade.

\subsection{Aplicação do questionário nas áreas definidas}

Segundo Günther (2003), um questionário pode ser aplicado de diferentes formas: entrevista individual ou por telefone, através de interação pessoal; ou enviado via correio. Métodos mais modernos incluem os chamados questionários online, que são enviados via e-mail ou aplicativos de redes sociais.

Neste trabalho, buscando alcançar uma amostra bastante variada, sugere-se que o questionário seja distribuído em escolas públicas das duas localidades selecionadas, de diferentes níveis de formação escolar (pré-escola, ensino fundamental, médio e técnico, se houver). A escola enviará os questionários para os familiares através da agenda escolar do aluno. Os familiares preencherão o questionário dentro de um prazo especifico e o retornarão para a escola pela mesma forma. Este método de pesquisa é conhecido como Deixar/Buscar (Drop-off / Pick-up), e costuma apresentar taxas de resposta mais altas que outros métodos (Jackson-Smith et al., 2016). Outra vantagem deste formato é a padronização, mais flexível em entrevistas pessoais. Caso não seja alcançado o número de respondentes mínimo determinado pela amostra mínima, alguns questionários podem ser aplicados em lojas e farmácias do comércio local, como um complemento.

\subsection{Tabulação dos dados obtidos}

A pesquisa quantitativa, de acordo com Marconi e Lakatos (2011), deve ter sua ênfase na quantificação das suas componentes, de forma metódica e objetiva.

As respostas de todas as questões propostas nos questionários preenchidos podem ser quantificadas, permitindo que se trace um perfil atualizado do comportamento das populações observadas. Como resultados imediatos sobre a retenção de REEE nos domicílios, espera-se quantificar as seguintes variáveis: 
- quantos respondentes (\%) retém aparelhos eletroeletrônicos sem uso em casa;

- uma estimativa da quantidade de REEE atualmente retida;

- uma estimativa do tempo médio de retenção destes REEE.

Nos países em desenvolvimento muitas vezes as pessoas se declaram ambientalmente conscientes, porém não apresentam conhecimento realmente consistente sobre o assunto (Zhao et al., 2014), especialmente se não houver algum tipo de incentivo, como retorno reembolsável de um produto pós-consumo (Arslan, Yilmaz e Aksov, 2012). Esta consciência ambiental também não se traduz em ações de consumo consciente (Bekin, Carrigan e Szmigin, 2007; Echegaray e Hansstein, 2017). Neste contexto de conscientização ambiental e engajamento, o questionário proposto permite ainda coletar e quantificar informações mais detalhadas quanto ao comportamento no descarte, em face a dificuldades e preferências, tais como:

- quantos não descartam por falta de interesse ou quantos não descartam por falta de local apropriado próximo, por exemplo;

- quantos depositariam no lixo comum ou quantos colocariam na calçada, por exemplo.

- quantos efetivamente separam seu lixo reciclável.

Cada bairro estudado fornecerá seus dados para cada uma destas variáveis. Portanto, como resultado, ter-se-á uma extensa tabela com duas colunas de dados, que permitirão inicialmente uma análise comparativa/descritiva das diferenças e similaridades comportamentais de cada situação socioeconômica pesquisada.

Posteriormente, pode-se discutir os dados sob a ótica de uma cidade única, um intervalo cujos IDHs extremos seriam representados por estes dois universos pesquisados.

\subsection{Análise estatística dos dados}

Os resultados dos questionários, após a tabulação, deverão receber um tratamento estatístico, para validação e posterior análise e discussão dos resultados. Tal tratamento deve ser levado em consideração desde o momento de elaboração das perguntas do questionário. $O$ tratamento estatístico, de acordo com Ferreira (2018), pode ser efetuado utilizando-se programas de análise de dados existentes no mercado, como o Statiscal Packages for the Social Sciences por exemplo, ou outro similar.

As questões propostas no questionário serão do tipo em escala por intervalos, permitindo que se agrupe e classifique por intervalos proporcionais (Marconi e Lakatos, 2011). Cada pergunta, ou grupo de perguntas, gerará uma variável a ser validada e analisada.

\subsection{Considerações para posterior discussão dos resultados}

Nesta fase já se tem os resultados da pesquisa - as respostas dos questionários sobre as atitudes do consumidor frente ao descarte de REEE - devidamente tabulados e estatisticamente validados. Tais resultados deverão ser analisados através de um método analítico-sintético, ou seja, cada resultado será interpretado e discutido. Porém, o cenário completo, resultante da integração de cada parte, também será avaliado, visando expandir a compreensão sobre o comportamento das populações envolvidas frente ao descarte de REEE.

Uma análise comparativa/descritiva preliminar poderá ser efetuada para destacar as diferenças e similaridades de cada população pesquisada, no contexto socioeconômico de seu bairro (Marconi e Lakatos, 2011). Em seguida, é preciso analisar tais dados sob a perspectiva de um 
território contínuo, ou seja, a cidade do Rio de Janeiro e verificar se as informações coletadas permitem inferir um intervalo único, cujos IDHs extremos seriam representados por estes dois universos pesquisados.

Em um terceiro momento, os resultados deste estudo de caso poderão ainda ser comparados com outros resultados de pesquisas similares, objetivando expor analogias e discrepâncias. Estes estudos serão selecionados na fase de levantamento de dados bibliográficos. Echegaray e Hansstein (2017), por exemplo, estudou os procedimentos de descarte da população através de questionários. Diferentemente do presente estudo, a população estudada era das 9 principais cidades brasileiras e seu foco, porém, era identificar a distância entre a intenção em contribuir para a conservação do meio ambiente e o real comportamento adotado na prática, ou seja, no momento de reciclar ou de reduzir conscientemente o consumo.

\section{EXEMPLO DE APLICAÇÃO DA METODOLOGIA}

\subsection{Exemplo de definição dos locais pesquisados}

A título de exemplo, esta etapa da metodologia foi aplicada à cidade do Rio de Janeiro. A definição dos dois bairros considerou como fontes de dados duas fontes distintas.

A primeira é o relatório "Caracterização dos Resíduos Sólidos Domiciliares da cidade do Rio de Janeiro - 2016", elaborado pela Gerência de Pesquisas da Diretoria Técnica e de Logística da Companhia Municipal de Limpeza Urbana do Rio de Janeiro (COMLURB). Anualmente, a COMLURB disponibiliza em seu relatório, os resultados das análises gravimétricas dos resíduos urbanos coletados desde 1995 em cada rota de coleta na cidade. A partir de 2009, os dados referentes aos REEE passaram a ser discriminados no relatório.

A segunda fonte é o Atlas do Desenvolvimento Urbano no Brasil, conhecido como Atlas Brasil, elaborado pelo Instituto de Pesquisas Econômicas Aplicadas (IPEA), em conjunto com o Programa das Nações Unidas para o Desenvolvimento (PNUD), e a Fundação João Pinheiro (FJP), com apoio do Governo Federal. O Atlas Brasil é disponibilizado através de uma plataforma online, onde é possível realizar consultas ao IDH dos municípios brasileiros (IDH-M) e também ao IDH dos "bairros" de uma região metropolitana, de forma pontual ou como um ranking. Para o cálculo do IDH-M de cada município brasileiro e do IDH dos "bairros", os pesquisadores do IPEA, PNUD e FJP utilizam como base de dados os resultados dos Censos nacionais realizados a cada 10 anos pelo Instituto Brasileiro de Geografia e Estatística (IBGE). Os IDHs calculados pelo IPEA e PNUD são posteriormente validados pelo IBGE e, então, publicados no Atlas Brasil (Atlas Brasil, 2010).

No entanto, ao utilizar estas duas fontes de pesquisa, foi necessário adotar alguns critérios para garantir a correta representatividade da pesquisa. O relatório "Caracterização dos Resíduos Sólidos Domiciliares da cidade do Rio de Janeiro - 2016" apresenta dados sobre a quantidade de resíduos eletroeletrônicos presentes no lixo domiciliar coletado em 360 rotas de coleta ordinária, abrangendo quase todas as Regiões Administrativas. Neste relatório, os resultados de gravimetria são apresentados por bairros de cada Região Administrativa. No Atlas Brasil, a 
unidade territorial avaliada para o cálculo do IDH é chamada de "Unidade de Desenvolvimento Humano" (UDH), que pode ser um bairro, mas também um grupo de bairros ou uma subdivisão de um bairro.

De acordo com ranking do Atlas Brasil (2013), o melhor IDH do município do Rio de Janeiro é 0,959, na região próxima ao Parque Lage, no bairro Jardim Botânico, na Zona Sul da cidade; e o pior é 0,604, em locais tais como Vila Paciência e Jardim Água Branca, ambos na Zona Oeste da cidade.

Efetuando o cruzamento dos dados do relatório de gravimetria da COMLURB com os dados do Atlas Brasil correspondentes ao Ranking das Unidades de Desenvolvimento Humano do Rio de Janeiro, e eliminando distorções como subdivisões ou agrupamentos de bairros, é possível definir o bairro do Humaitá (IDH = 0,954), situado na Zona Sul da cidade, e o bairro de Barros Filho (IDH = 0,697), na Zona Norte, como os locais de IDH alto e baixo, respectivamente, a serem utilizados no estudo (COMLURB, 2017; Atlas Brasil, 2013).

A Figura 1 mostra a localização espacial de cada um dos bairros escolhidos. A distância direta entre eles é de $21,6 \mathrm{~km}$.

Figura 1 - Bairros do Humaitá e Barros Filho - referência espacial

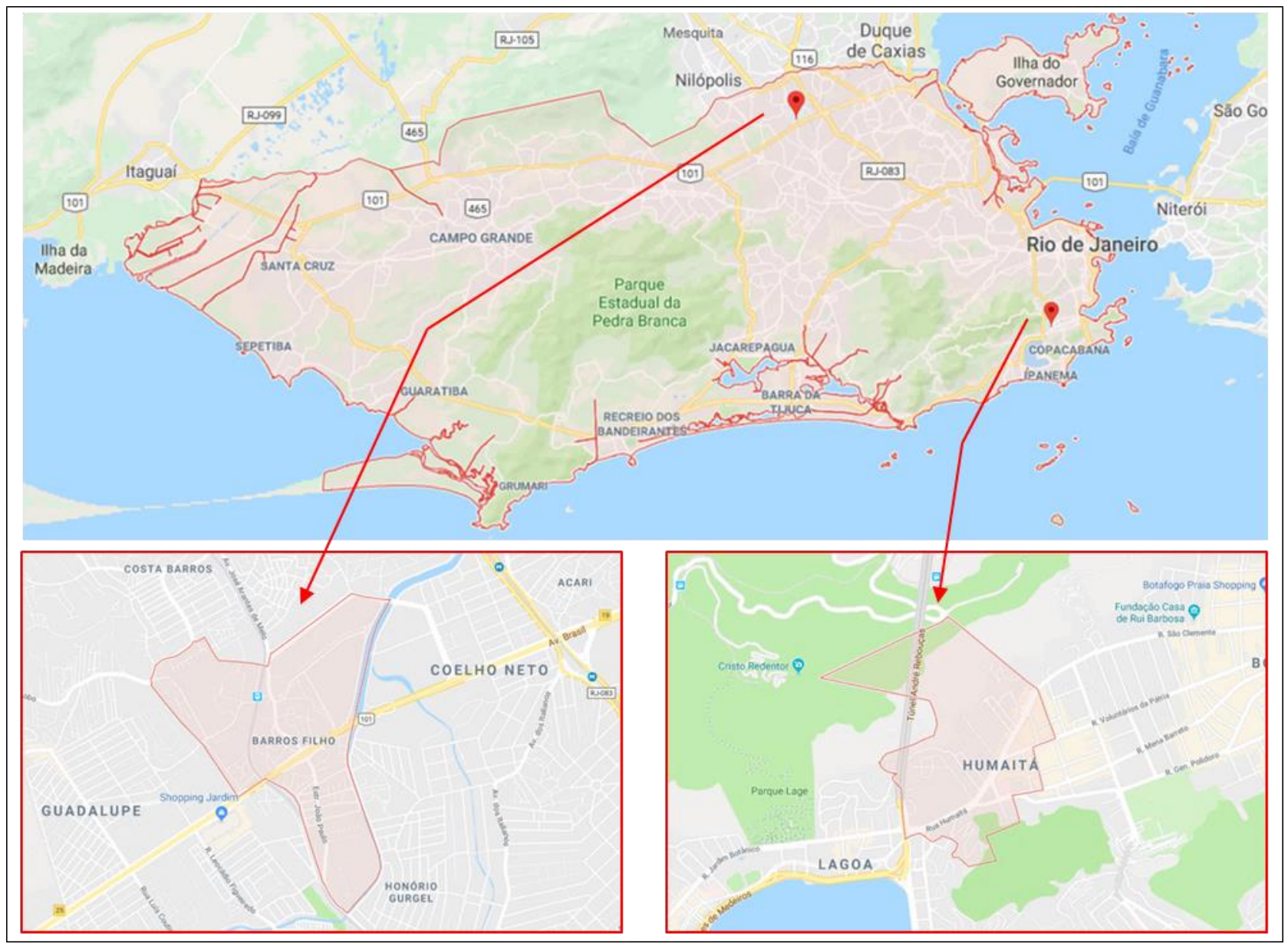

Fonte: Google Maps, 2018

Registros demográficos e geográficos sobre os bairros estudados deverão ser informados. No caso do Rio de Janeiro, tais informações foram obtidas a partir do Censo IBGE. Em 2010, o bairro 
do Humaitá possuía 13.285 habitantes distribuídos em uma área de 1,0682 km2, ou seja, 12.437,39 hab/km2. No mesmo ano, no bairro de Barros Filho, em uma área de 1,2352 km2, habitavam 7.919 pessoas, totalizando $6.411,32 \mathrm{hab} / \mathrm{km} 2$. A renda per capita do Humaitá era de $\mathrm{R} \$ 4.573,31 \mathrm{em} 2010$, enquanto que a de Barros Filho era de $\mathrm{R} \$ 553,70$.

\section{Dados sobre descarte inadequado de REEE em cada bairro}

Deve-se conhecer como o descarte do resíduo em questão é realizado nos dois bairros. Como exemplo, buscou-se informações na companhia de coleta de resíduos urbanos da cidade, no caso no relatório de gravimetria da COMLURB

Em 2016, no local com maior IDH foram coletadas $1.187 \mathrm{~kg}$ de amostras de resíduo domiciliar urbano, o chamado lixo comum das residências. Deste total, a COMLURB apurou que $0,47 \%$ eram REEE, ou seja, 5,58 kg. É possível, por proporcionalidade relativamente à população, assumir então que o bairro do Humaitá gerou $310 \mathrm{~g}$ de resíduo eletroeletrônico misturado aos resíduos comuns. Vale lembrar que o resíduo domiciliar comum - e tudo que acaba se juntando a ele - segue diretamente para o aterro sanitário da cidade e, portanto, este procedimento não constitui uma forma de descarte ambientalmente adequada. Apesar do Humaitá ser um bairro com alto nível de renda e educação, ainda apresenta dificuldades no momento do descarte, que pode advir de diversas causas, como desinformação ou desinteresse, por exemplo.

Em 2016, no bairro de Barros Filho (bairro com menor IDH), foram coletadas $656 \mathrm{~kg}$ de amostras de resíduo domiciliar urbano. Deste total, a COMLURB apurou que 0,78\% eram REEE, ou seja, $5,1 \mathrm{~kg}$. Pode-se, por proporcionalidade relativa à população, assumir então que o bairro de Barros Filho gerou $344 \mathrm{~g}$ de resíduo eletroeletrônico misturado aos resíduos comuns, praticamente o dobro do percentual calculado para Humaitá.

Vários fatores podem influenciar e explicar esta conduta, até mesmo uma combinação dos mesmos. No caso deste estudo, é importante verificar, durante a análise estatística dos dados, qual é o grau de correlação entre a variável IDH e o descarte inadequado de REEE.

\section{Dados sobre a evolução do IDH em cada bairro}

Deve-se conhecer o comportamento do IDH dos bairros estudados, a fim de ampliar a compreensão sobre o perfil da população local. Segundo o Atlas Brasil, o bairro do Humaitá apresentou evolução no IDH entre 2000 e 2010, evoluindo de 0,899 para 0,954 no período. 0 bairro de Barros Filho, por sua vez, apresentou uma evolução em seu IDH de 0,566 para 0,697 entre os Censos de 2000 e 2010. A Figura 2 mostra esta evolução de cada um dos três componentes do IDH-M nos dois bairros.

Figura 2- Evolução dos Componentes do IDH-M dos bairros do Humaitá e de Barros Filho 


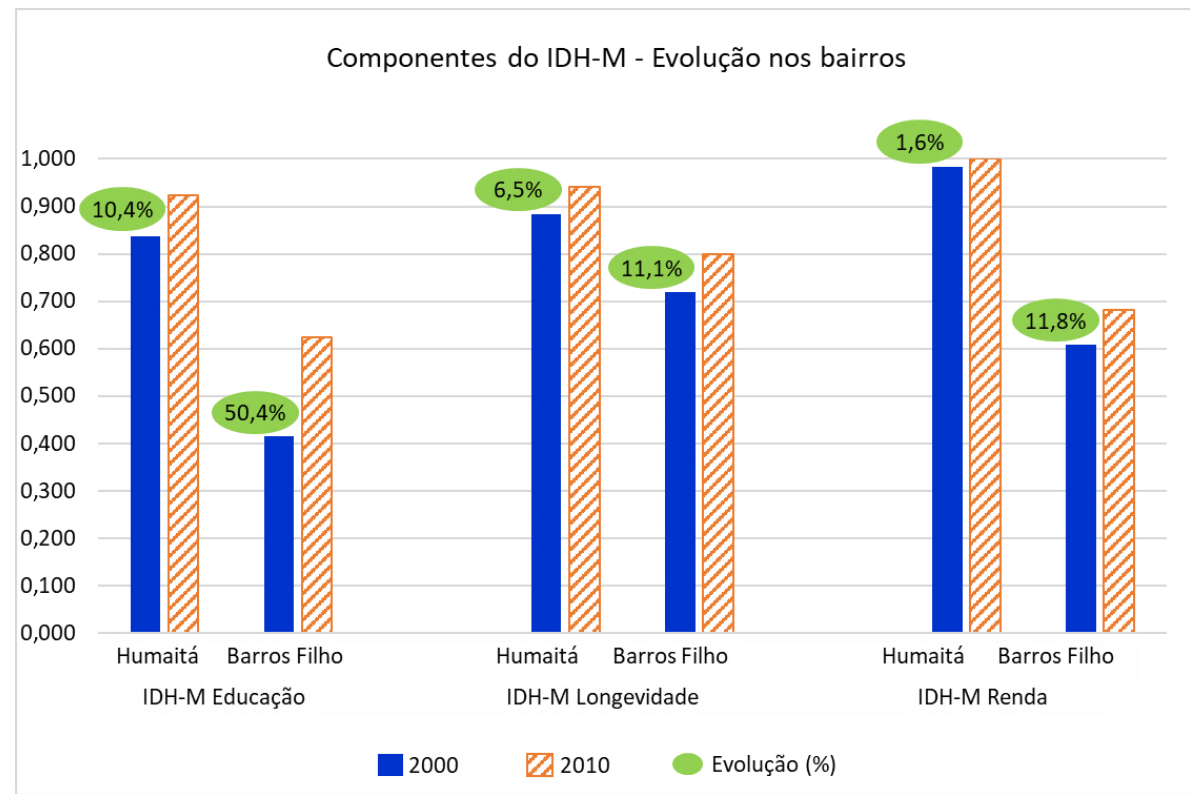

Fonte: Atlas Brasil, 2010 / Elaboração dos autores

Observa-se que a componente de Educação foi a que apresentou maior crescimento no período, influenciando positivamente de forma bastante relevante na evolução do IDH-M de ambos os bairros. Esta característica foi ainda mais expressiva no bairro de Barros Filho. Esta constatação é importante, pois o grau de conscientização sobre resíduos é, usualmente, diretamente relacionado ao nível geral de educação de uma população.

\subsection{Exemplo de definição do questionário}

A título de exemplo, aplicando-se a metodologia apresentada, desenvolveu-se um modelo de questionário, cujo conteúdo ainda deve ser validado antes de sua efetiva aplicação nas áreas definidas. O modelo é apresentado na Figura 3. 
Figura 3 - Questionário preliminar

EQUIPAMENTOS ELETROELETRÔNICOS de pequeno porte são, por exemplo:

- telefone celular

- rádio

- telefone sem fio

- videogame

- notebook

- aparelho de fax
- controle remoto

- televisão / monitor

- secador / chapinha

- microondas

- liquidificador

- furadeira
- caixa de som

- aparelho de CD / DVD

- impressora / cartucho

- brinquedo com som / luz

- ferro de passar / aspirador

- cabos, etc.

\section{PESQUISA}

1) Você tem, guardado em sua casa, algum desses equipamentos eletroeletrônicos com defeito ou que você não usa mais?
( ) Nenhum
( )
( ) 2
( ) 3
( ) 4
( ) 5 ou mais

2) Por quanto tempo você costuma guardar estes aparelhos eletroeletrônicos?
( ) 3 meses
( ) 6 meses
( ) 1 ano
( ) 2 anos ou mais

3) Por que ainda não descartou seus aparelhos eletroeletrônicos?
( ) Não tenho interesse.
( ) Eles ainda funcionam.
( ) Eles ainda contém informações que posso precisar.
( ) Tenho medo que usem minhas informações particulares.
( ) Não sei onde levar.
( ) Outros:

4) Como você descartaria seus aparelhos eletroeletrônicos?

( ) Colocaria na lixeira comum.

( ) Chamaria o serviço de remoção gratuita da COMLURB.

( ) Colocaria na calçada da minha rua.

( ) Entregaria para o carro do ferro-velho que passa na rua.

( ) Colocaria no coletor para eletroeletrônicos mais próximo.

( ) Outros:

5) Onde seria o local mais adequado para a instalação de um Ponto de Coleta para descartar seus aparelhos eletroeletrônicos?

( ) Na farmácia, supermercado ou escola mais próxima.

( ) Em alguma das estações da Supervia ou do Metrô.

( ) Outra sugestão:

6) Você costuma separar o lixo reciclável na sua casa (plástico / vidro / papel / metal)?
( ) Nunca.
( ) Às vezes
( ) Sempre

OBRIGADO!

Fonte: Elaboração dos autores

\section{CONSIDERAÇÕES FINAIS}

A participação do consumidor é considerada, em muitos países, fator determinante para o sucesso da logística reversa de resíduos de equipamentos eletroeletrônicos. Neste contexto, o objetivo deste trabalho foi apresentar uma metodologia para identificação e análise dos procedimentos do consumidor urbano quanto ao descarte deste tipo de resíduo, na qual fosse 
considerado não apenas o viés econômico, mas também características sociais. Para tal, a metodologia sugere a aplicação de questionário impresso a duas amostras populacionais de uma mesma cidade, porém com características socioeconômicas diferentes, determinadas pelo cruzamento de dados da empresa pública de limpeza urbana e de um ranking de IDH dos bairros da cidade.

Através desta metodologia, após a definição das áreas de pesquisa, aplicação do questionário, tabulação e análise dos dados obtidos, será possível traçar um panorama dos procedimentos adotados atualmente pelos consumidores quanto ao descarte dos REEE. A metodologia permite ainda ampliar o conhecimento sobre a cadeia de REEE no contexto urbano, propiciando o desenvolvimento de sugestões de ações e políticas públicas que possam aumentar a probabilidade de retorno dos REEE ao setor produtivo, em linha com a economia circular.

\section{AGRADECIMENTOS}

Os pesquisadores agradecem as contribuições técnicas do projeto "Latin American-European network on waste electrical and electronic equipment research, development and analyses" (LaWEEEda), financiado pelo fundo Erasmust, e ao CNPq e à FAPERJ o constante apoio recebido.

\section{REFERÊNCIAS BIBLIOGRÁFICAS}

AIZAWA, Hirofumi; YOSHIDA, Hideto; SAKAI, Shin-ichi. Current results and future perspectives for Japanese recycling of home electrical appliances. Resources, Conservation and Recycling 52, 2008, p. 1399-1410. DOI: 10.1016/j.resconrec.2008.07.013

ARSLAN, T.; YILMAZ, V.; AKSOV, H.K. Structural equation model for environmentally conscious purchasing behavior. Int. J. Environ. Res. 6, 2012, p. 323-334. DOI: 10.22059/IJER.2011.498.

ATLAS BRASIL. Atlas do Desenvolvimento Urbano no Brasil. Brasil, 2013. Disponível em: http://www.atlasbrasil.org.br. Acesso em: 12 de agosto de 2018.

BALDÉ, C.P., WANG, F., KUEHR, R., HUISMAN, J. The global e-waste monitor - 2014, United Nations University (UNU), IAS - SCYCLE, Bonn, Germany, 2015. ISBN: 978-92-808-4555-6

BALDÉ, C.P.; FORTI V.; GRAY, V.; KUEHR, R.; STEGMANN, P. (2017). The Global E-waste Monitor - 2017, United Nations University (UNU), International Telecommunication Union (ITU) \& International Solid Waste Association (ISWA), 2017. ISBN: 978-92-808-9053-2

BEKIN, Caroline; CARRIGAN, Marylyn; SZMIGIN, Isabelle. Beyond recycling: “commons-friendly" waste reduction at new consumption communities. Journal of Consumer Behavior. 6, 2007, p. 271-286. DOI: 10.1002/cb.221

BERNARDO, Marcella; LIMA, Renato. Comparação entre Modalidades de Coleta Seletiva de Materiais Recicláveis. XXIX Congresso Nacional de Pesquisa em Transporte da ANPET, Transporte e Meio Ambiente II, Aspectos Econ. Soc. Políticos e Ambientais do Transporte, 2015, p. 2497 - 2507.

BIROCHI, R. Metodologia de Estudo e de Pesquisa em Administração Pública. Programa Nacional de Formação em Administração Pública (PNAP). Especialização em Gestão Pública, Módulo Básico, 2015. ISBN: 978-85-7988-262-3.

BRASIL. Lei Federal № 12.305, de 2 de agosto de 2010. Política Nacional de Resíduos Sólidos, 2010. Disponível em: http://www.planalto.gov.br/ccivil 03/ ato2007-2010/2010/lei/l12305.htm. Acesso em: 04 de julho de 2018.

COMLURB. Caracterização dos Resíduos Sólidos Domiciliares da cidade do Rio de Janeiro - 2016. Gerência de Pesquisas, Diretoria Técnica e de Logística, Companhia Municipal de Limpeza Urbana do Rio de Janeiro - COMLURB, 2017. 
DARBY, Lauren; OBARA, Louise. Household recycling behaviour and attitudes towards the disposal of small electrical and electronic equipment. Resources, Conservation and Recycling 44, 2005, p. 17-35. DOI: 10.1016/j.resconrec.2004.09.002

DIAS, Pablo; MACHADO, Arthur; HUDA, Nazmul; BERNARDES, Andréa Moura. Waste electric and electronic equipment (WEEE) management: A study on the Brazilian recycling routes. Journal of Cleaner Production 174, 2017, p. 7-16.DOI: 10.1016/j.jclepro.2017.10.219

ECHEGARAY, Fabian.; HANSSTEIN, Francesca. Assessing the intention-behavior gap in electronic waste recycling: the case of Brazil. Journal of Cleaner Production 142, 2017, p. 180-190. DOI: 10.1016/j.jclepro.2016.05.064

EUROPEAN UNION. Directive 2012/19/EU of the European Parliament and of the Council of 4 July 2012 on waste electrical and electronic equipment (WEEE). Official Journal of the European Union, 24.07.2012 - EN - L 197/38. Disponível em: https://eur-lex.europa.eu/legal-content/EN/TXT/?uri=CELEX:32012L0019. Acesso em: 18 de agosto de 2018. DOI:10.3000/19770677.L_2012.197.eng

FERREIRA, Jhony; PACHECO, Elen; SILVA, Ana Lúcia. Uma Proposta para Identificação de Indicadores de Sustentabilidade para Avaliação do Gerenciamento de Resíduos Eletroeletrônicos com Foco na Reciclagem. Revista Gestão e Gerenciamento № 10, 2018, p. 18-26.

GENG, Yong; DOBERSTEIN, Brent. Developing the circular economy in China: Challenges and opportunities for achieving 'leapfrog development'. The International Journal of Sustainable Development \& World Ecology, 15:3, 2008, p. 231-239. DOI: 10.3843/SusDev.15.3:6

GHISELLINI, Patrizia; CIALANI, Catia; ULGIATI, Sergio. A review on circular economy: the expected transition to a balanced interplay of environmental and economic systems. Journal of Cleaner Production 114, 2016, p. 11-32. DOI: 10.1016/j.jclepro.2015.09.007

GÜNTHER, Hartmut. Como Elaborar um Questionário. Série: Planejamento de Pesquisa nas Ciências Sociais, № 01. Brasília, DF: UnB, Laboratório de Psicologia Ambiental, 2003. Disponível em: http://www.psiambiental.net/pdf/01Questionario.pdf. Acesso em 16 de abril de 2019.

HOSS, Marcelo; CATEN, Carla. Processo de Validação Interna de um Questionário em uma Survey Research Sobre ISO 9001:2000. Produto \& Produção, vol. 11 n. 2, 2010, p. 104 - 119. DOI: 10.22456/1983-8026.7240

JACKSON-SMITH, Douglas et al. Effectiveness of the Drop-off-Pick-up Survey Methodology in Different Neighborhood Types. Journal of Rural Social Sciences, 31(3), 2016, pp. 35-67.

MARCONI, Marina; LAKATOS, Eva Maria. Metodologia Científica. 6a Edição. 2011. Editora Atlas.

NEUMAYER, Eric. The human development index and sustainability - a constructive proposal. Ecological Economics 39, 2001, p. 101-114. Elsevier. DOI: 10.1016/S0921-8009(01)00201-4

NNOROM, I.C.; OHAKWE, J.; OSIBANJO, O. Survey of willingness of residents to participate in electronic waste recycling in Nigeria - A case study of mobile phone recycling. Journal of Cleaner Production 17, 2009, p. 1629-1637. DOI: 10.1016/j.jclepro.2009.08.009.

RAMALHO, Ângela; MARQUES, Francisca. Classificação da pesquisa científica. UFRN/UEPB. 2009.

RODRIGUES, Angela; GUNTHER, Wanda; BOSCOV, Maria Eugenia. Estimativa da geração de resíduos de equipamentos elétricos e eletrônicos de origem domiciliar: proposição de método e aplicação ao município de São Paulo, São Paulo, Brasil. Revista Engenharia Sanitária e Ambiental, v.20 n.3, p. 437-447. DOI: 10.1590/S1413-41522015020000133701.

SINIR. Logística Reversa. Sistema Nacional de Informações sobre Resíduos Sólidos - SINIR, do Ministério do Meio Ambiente - MMA. Disponível em: https://sinir.gov.br/logistica-reversa. Acesso em 01 de agosto de 2019.

SOUZA, Maria Tereza; PAULA, Mabel; SOUZA-PINTO, Helma. O papel das cooperativas de reciclagem nos canais reversos pós-consumo. FGV-EAESP. Revista de Administração de Empresas, vol. 52, n. 2, 2012. DOI: $10.1590 /$ S0034-75902012000200010

SU, Biwei; HESHMATI, Almas; GENG, Yong; YU, Xiaoman. A review of the circular economy in China: moving from rhetoric to implementation. Journal of Cleaner Production 42, 2013, p. 215-227. DOI: 10.1016/j.jclepro.2012.11.020 
UNDP. Human Development Report 2016 - Human Development for Everyone. United Nations Development Programme. 2016. ISBN: 978-92-1-126413-5.

YIN, Jianfeng; GAO, Yingnan; XU, He. Survey and analysis of consumers' behaviour of waste mobile phone recycling in China. Journal of Cleaner Production 65, 2014, p. 517e525. DOI: 10.1016/j.jclepro.2013.10.006

ZHAO, Hui-hui et al. What affects green consumer behavior in China? A case study from Qingdao. Journal of Clean Production. 63, 2014, p. 143-151. DOI: 10.1016/j.jclepro.2013.05.021

ZHIJUN Feng; NAILING, Yan. Putting a circular economy into practice in China. Sustain Sci v.2, 2007, p. 95-101. DOI: $10.1007 / \mathrm{s} 11625-006-0018-1$ 